\title{
The Design of Portable Somatosensory Exercise Bike based on Single Chip Microcomputer
}

\author{
Liu Jingchao \\ School of Control Engineering \\ Xijing Universuty \\ Xi'an, China \\ 805444342@qq.com
}

Cao Yanyan

Network Security and Data Department

Xijing Universty

Xi'an, China

e-mail: cyy647@126.com

\author{
$\mathrm{Li} \mathrm{Na}$ \\ Academy of Arts \\ Xijing Universuty \\ Xi'an, China \\ 1353632387@qq.com \\ Cai Hongzhuan \\ School of Control Engineering \\ Xijing Universuty \\ Xi'an, China \\ 175534337@qq.com
}

\begin{abstract}
As the increasing of residents' income and the improvement of living standards, the number of mass entertainment increased. With its growth, the pressure of competition is becoming more and more obvious. Because of the high pressure working mode, a lot of people are in the sub-health state. They urgently need a kind of fitness machine that can combine entertainment and fitness. To address the problem, the designer adopt the man-machine interaction pattern, and invented a kind of fitness with game consoles. This paper introduces such an exercise bike to meet the requirements. The designer use power electronic technology to convert ordinary bicycle into a stationary bike. At the same time, the bike also has the function of entertainment. It is connected to the game through the computer. When people use it, they make themselves in the game scene by a computer screen or a curtain. The bike uses the technology of single-chip microcomputer, and is made of very portable. It enhances the fitness fun. The experiment proving that this is achievable.
\end{abstract}

Keywords-Single chip microcomputer; Exercise bike; Somatosensory; Portable; man-machine interaction

\section{THE DESIGN BACKGROUND}

As the increasing of residents' income and the improvement of living standards, the number of mass entertainment increased. With its growth, the pressure of competition is becoming more and more obvious. Because of the high pressure working mode, a lot of people are in the sub-health state. Because of the busy work and the limited spare time, it is an urgent need to combine sports and entertainment, to achieve the goal of sports-entertainment.

In the present market, there is an equipment that meets the above requirements, Such as 3D tennis game machine. But their price is usually more expensive, and there is a large part of the 3D device, not easy to move. In ordor to make the user convenience, and to facilitate the movement of the sports, and reduce the cost, this paper introduces a kind of portable dynamic experience fitness bike device design.

\section{SYSTEM OVERALL FRAME DESIGN}

This design is made up of tree parts, bicycle, electronic equipment and connecting part. It was installing electronic devices on a regular cycle. Then it was connected with the electronic equipment through the connecting device. The electronic equipment can be a computer or a television. The fitness bike user can gym in the game environment through these devices. You also can say that this is a kind of entertainment. In the process of entertainment, you can exercise your body.

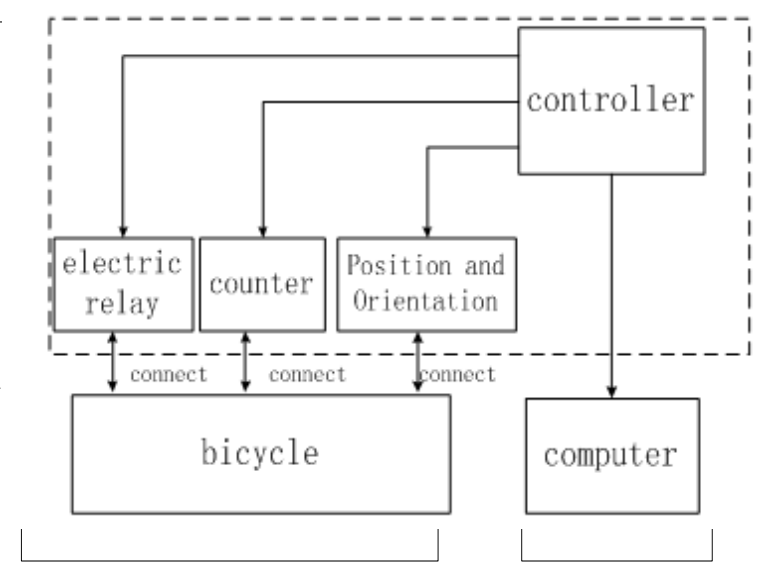

Figure 1. System overall frame diagram.

As shown in Fig .1, the connecting part includes electric relay, counter and position and orientation. The electric relay is used to control the opening of the line. The counter is used to calculate the number of gears traversed. Then the number of gears converted to the speed. Position and Orientation is used to obtain direction information and transform it into the game. They get the movementi nformation of the bicycle and 
react them to the game through the 51 microcontroller. When the bicycle handlebar turn to left, the car is left in the game. When the bicycle handlebar turn to right, the car is right in the game. When people ride faster, the faster the car in the game.

That is to say people ride on a bicycle. The cycling information is displayed in the game. This is a wonderful experience.

\section{RELATED TECHNICAL DESCRIPTION}

\section{A. Open Cobalt}

Open Cobalt is a multi-user virtual environment that comes from the free and open source browser for virtual work and construction of the toolkit to access. And it creates and publishs a multi-user.

This station provides a common participant by croquet technology, open cobalt using command group communication protocol. It continuous copying by multi guest virtual work a real-time dynamic calculation, without virtual world server. This also makes it very simple to create and securely sharing collaborative virtual work that is deeply run on all major software operating systems.

This is the author's development kit.

\section{B. Clanguage}

$\mathrm{C}$ language is a computer programming language. It has the characteristics of high-level language, but also has the characteristics of assembly language. It was launched in 1972 by the American Institute of Baer's D.M.Ritchie, and after 1978, the C language has been ported to large, medium, small and micro machines. It can be used as a working system design language, writing system applications, and can also be used as an application design language, writing application program does not relying on the computer hardware. Its wide range of applications, with strong data processing capabilities, make $\mathrm{C}$ language not only be used in the software development, but also in all kinds of scientific research. It is suitable for writing system software, three-dimensional, two-dimensional graphics and animation, specific applications such as single-chip microcomputer and embedded system development.

$\mathrm{C}$ language file is composed of data sequence. $\mathrm{C}$ language IDE that can constitute a binary file or text file commonly useing are as follow. $\mathrm{C}++, \mathrm{C}++$ Watcom Borland Borland Code, C++ GNU, C++ Builder, DJGPP C++, C Compiler Mac, Lccwin32 High, C Turbo, C x, OS C-Free, win-tc Blocks, Dev-C++, Microsoft, xcode.

\section{Single chip microcomputer}

The full name of the Single is a single-chip microcomputer. In order to use it conveniently, it integrates the main functional components of the computer at a semiconductor chip, such as the central processor(CPU), data storage(RAM), program memory(EPROM, E2PROM, ROM or FLASH), timing /counter and various input/output interface circuits. It constitutes a complete computer system.

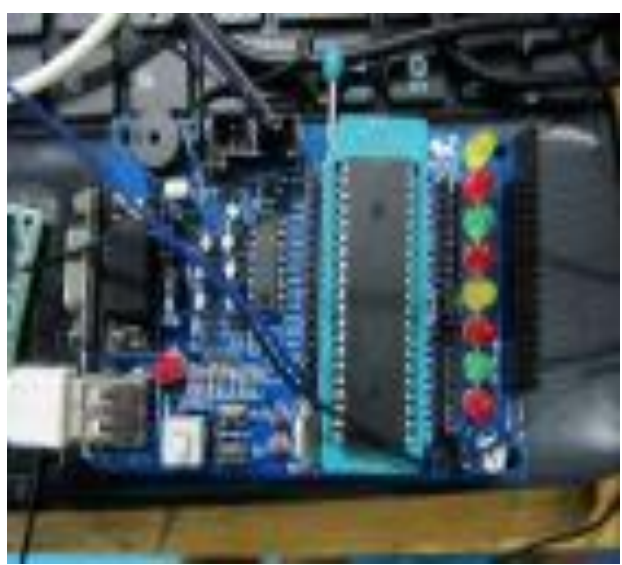

Figure 2. 51 microcontroller development board.

The instruction function of the single-chip microcomputer is designed according to the requirements of industrial control, so it is also called the microcontroller (Microcontroller).

\section{Single chip microcomputer application system}

The smallest single-chip microcomputer application system circuit refers to the most simple circuit which can work normally. AT89S51 microcontroller minimum application system circuit diagram shown in Fig .2.

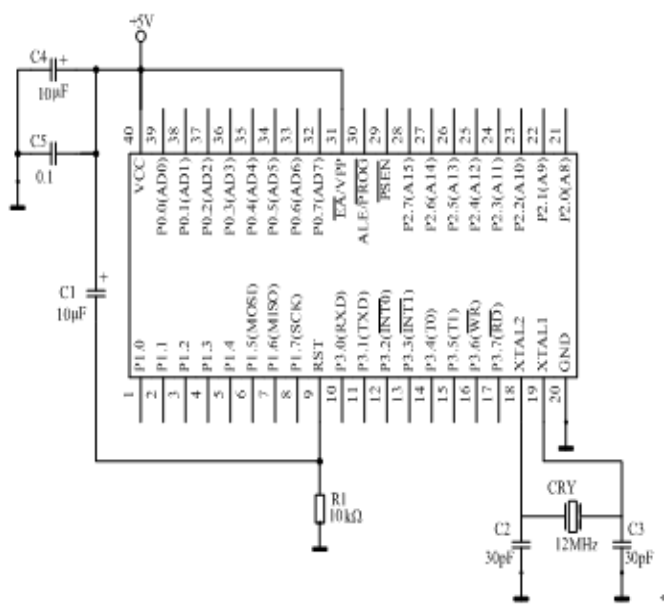

Figure 3. AT89S51 minimum application system diagram.

The system contains 4 parts.

- Power supply circuit. Pin VCC (pin 40) is connected to the $+5 \mathrm{~V}$ power supply. Pin GND (pin 20 ) is connected to the ground wire. In order to improve the anti-interference ability of the circuit, a $0.1 \mathrm{uF}$ (labeled as 104 devices) electrolytic capacitor of $\mathrm{F}$ ceramic capacitor and a $10 \mathrm{uF}$ capacitor is connected between the VCC pin and ground.

- The program selected memory circuit. As mentioned before, the Atmel 8051 compatible chip has a variety of internal program memory, so it does not need to be extended to the use of external program memory, so that the microcontroller 
application circuit in the pin (pin 31) can always be high.

- Clock circuit. The clock frequency of the AT89S51 chip can be in the range of $0 \sim 33 \mathrm{MHz}$. Single chip microcomputer has an amplifier circuit that can constitute oscillator. It can form a single chip clock circuit by connecting the crystal and the capacitor between the external pin XTAL2 (pin 18) and XTAL1 (pin 19) In this amplifying circuit. The clock frequency of the chip is determined by the frequency of the crystal CRY. If the bread board is used to assemble the single chip application circuit, the recommended value of the crystal CRY is below $12 \mathrm{MHz}$. The value of the capacitor $\mathrm{C} 2$ and $\mathrm{C} 3$ is from $30 \mathrm{pF}$ to $50 \mathrm{pF}$. The purpose of the clock circuit is to increase the stability of the clock frequency.

- Reset circuit. For the AT89S51 chip, if the pin RST (pin 9) maintains a high level of 24 clock cycles, the microcontroller can complete the reset. Usually in order to ensure the application of the system to be reliable reset, the reset circuit should be kept above the RST level of 10ms. As long as the RST pin remains high, the microcontroller will reset the cycle. When the pin RST is changed from a high level to a low level, the single chip microcomputer is out of the reset state, and the $0000 \mathrm{H}$ address of the program space begins to execute the user program.

\section{E. The counter}

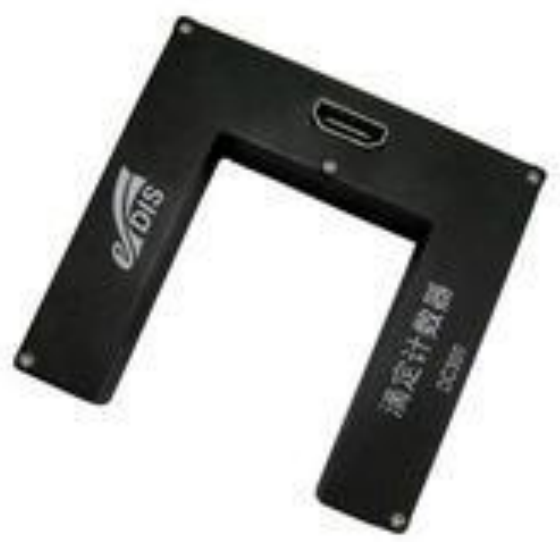

Figure 4. The counter.

Counting is one of the most simple and basic operation. the counter is to realize the logic circuit. Counter in the digital system is mainly to count the pulse of a number of functions, in order to achieve measurement, counting and control functions. At the same time, the counter is composed of basic counting unit and some control door. Counting unit is made up of various types of triggers that have memory information functions. Such as RS trigger, T trigger, D trigger and JK trigger. Counter is widely used in digital systems, such as in the electronic computer controller to count the instruction address, in order to take out the next instruction.

\section{F. The electric relay}

Electric Relay (English Name: Relay) is a kind of electric control device, which is a kind of electrical apparatus that can make the step change of the controlled quantity in the electrical output circuit when the input quantity (amount of excitation) change to reach the specified requirement. It has a control system (also known as the input loop) and the interaction between the control system (also known as the output circuit). It is usually applied to an automatic control circuit, which is in fact an automatic switch to control the operation of large current with small current. Therefore, in the circuit it plays an automatic adjustment, safety protection, switching circuit and so on.

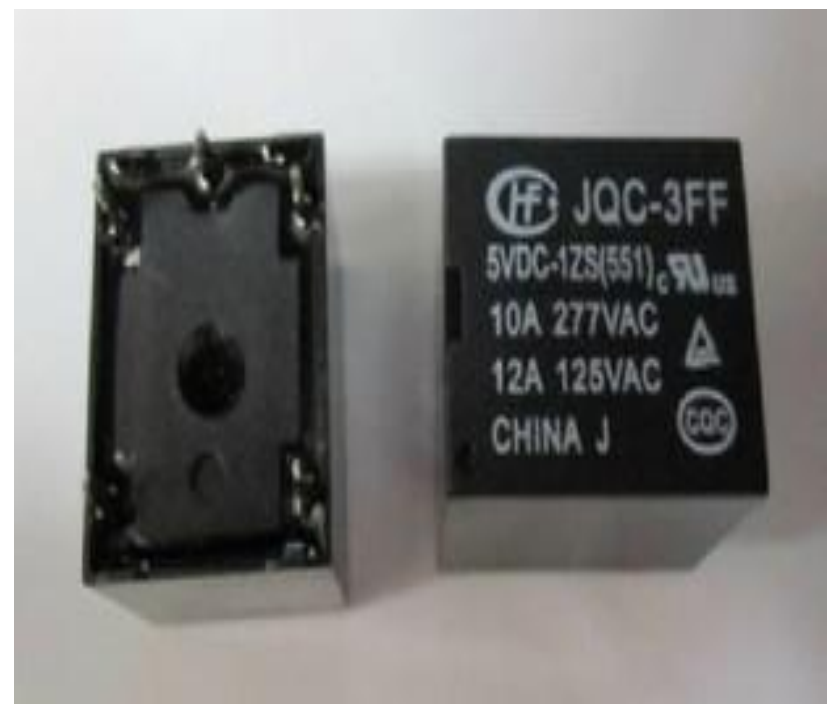

Figure 5. The electric relay.

The general electromagnetic relay is composed of an iron core, coil, armature, contact etc.. As long as the coil ends with a voltage, the current will flow through the coil, resulting in electromagnetic effects, the armature will attract the electromagnetic force to overcome the spring to return to the core rally absorption, thereby driving the armature static and dynamic contact (normally open). When the coil power off, the electromagnetic force disappears, the armature will return to the original position in the spring reaction, the dynamic contact with the original static contact (normally closed contact) release. This pull and release, in order to achieve the circuit in conducting or cutting purposes. For the relay, normally open and normally closed contact, can be distinguished as follow: the relay coil is not energized when the static contact, called "normally open contact" ". The relay has two circuits, which is a low voltage control circuit and a high voltage circuit.

\section{THE EXERCISE BICYCLE}

The designer places the exercise bicycle in the scene of the game by using science and technology. 
It mainly completes the the game handle simulation. It runs on the direction sensor, the counter implementation, the finishing direction sensors and counter assembly.



Figure 6. User on the exercise bicycle.

As shown in Fig .6 and Fig .7, the exercise bicycle can be very small and the Movement process can be very interesting.

This is just an example. This design can also be used for other types of bikes. And you can also use other game scenario.

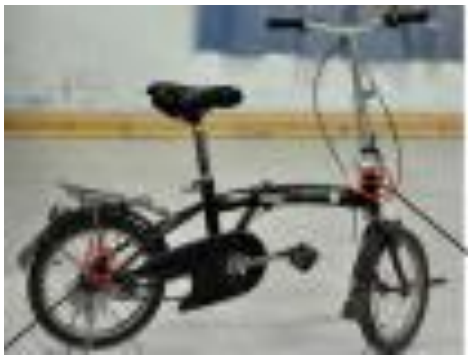

Figure 7. The exercise bicycle.

\section{THE ADVANTAGES OF THE EXERCISE BICYCLE}

There are many benefits of using the exercise bicycle, specific as follows.

- Bicycle is one of the best tools to overcome the problem of heart function. More than half of the world's people die of heart disease, the movement of a bicycle can compress blood flow through the legs, and blood from the blood vessels to the heart, in fact, it has also strengthened the microvascular tissue, which is called collateral circulation.

- At the same time, Exercisecan can prevent hypertension, sometimes more effective than drugs. It can prevent weight gain, blood vessel sclerosis, and make bone strong. Bicycles make you don't have to use drugs to keep healthy.

- Bicycle is a tool for weight loss. According to statistics, people of $75 \mathrm{~kg}$ weight, riding 73 miles with 9 miles per hour speed, can reduce the weight of half a kilogram, but must be consistent every day.

- Riding a two wheeled bicycle which rely on the physical strength to step on, you will feel very free and very fun. It is not only a kind of weight loss exercise, but also a spiritual pleasure.

\section{CONCLUSIONS}

According to the survey, the current people's sense of fitness has been fully consolidated. While the single function, lack of entertainment and interaction of fitness equipment, has been unable to meet the people's pursuit of high-quality fitness.

In this paper, the research and development of fitness equipment embedded system based on distributed virtual environment is to solve this problem and produce. The research of distributed virtual environment has become a hotspot in computer graphics research. It has the characteristics of multi-sensation, immersion, interactivity, imagination, etc.. With the rapid development of computer hardware and greatly reduce the cost, this technology is widely used in medical, construction,manufacturing, entertainment, sports, military and other fields. The research and development of fitness equipment embedded system based on distributed virtual environment is an attempt to apply the virtual environment technology to the field of fitness equipment. The system with the fitness equipment as application object, with the help ofcomputer, sensor, multimedia, network communication technology, through the network connection, support for multiple users to interact in virtual scenes.

\section{REFERENCES}

[1] Recent progress in single chip white light-emitting diodes with the InGaN underlying layer[J]. Science China(Physics,Mechanics \&amp; Astronomy),2010,03:445-448.

[2] Wang Qingmin, Yang Yaoen. Teaching Reform of the Course of Single Chip Microcomputer[A]. Atlantis Pres. Beijing xin yongshun culture communication co., LTD. Proceedings of 2013 International Conference on Information,Business and Education Technology(ICIBET 2013)[C].Atlantis Press 、 Beijing xin yongshun culture communication co., LTD:,2013:4.

[3] Fuyan Zhang,Chunjun Zheng. Research on Single Chip Computer Teaching Reform under CDIO Engineering Education Mode[A]. Computer science and electronic technology international society:,2013:4.

[4] Ruan Yue,Yao Wenji,Tang Ying. Design of a single chip radio transceiver based on SoC[A]. Intelligent Information Technology Application Association.Proceedings of 2011 International Conference on Computers, Communications, Control and Automation Proceedings (CCCA 2011 V3) :,2011:4.

[5] The Application of Multitasking Mechanism in Single Chip Computer System[J]. Wuhan University Journal of Natural Sciences, 1999,01:59-62.

[6] SF VECTOR CONTROL SYSTEM WITH TWO SINGLE CHIP MICROPROCESSORS[J]. Journal of China Textile University(English Edition),1990,02:73-78.

[7] Jun Li,Xian-Lin Meng,Wen-Long Song. Intelligent Temperature Control System Design Based on Single-Chip Microcomputer[J]. Journal of Harbin Institute of Technology,2014,03:91-94.

[8] Xin Zhou. Electronic truck scale design based on 51 single chip microcomputer[D].Nanjing University of Science and Technology,2014.

[9] Xiaohai Tao. 51 singlechip C language programming skills[J]. Computer generation, 2009,06:41-42.

[10] Xiaoli An. The design of the AT89S51 development board[J]. Electronic Design Engineering,2009,09:121-123. 